\title{
Covariance Matrix Repairing in Gaussian Based EDAs
}

\author{
Weishan Dong, and Xin Yao, Fellow, IEEE
}

\begin{abstract}
Gaussian models are widely adopted in continuous Estimation of Distribution Algorithms (EDAs). In this paper, we analyze continuous EDAs and show that they don't always work because of computation error: covariance matrix of Gaussian model can be ill-posed and Gaussian based EDAs using full covariance matrix will fail under specific conditions. It is a universal problem that all existing Gaussian based EDAs using full covariance matrix suffer from. Through theoretical analysis with examples of simulated data and experiments, we show that the ill-posed covariance matrix strongly affects those EDAs. This paper proposes a Covariance Matrix Repairing (CMR) method to fix ill-posed covariance matrix. CMR significantly improves the robustness of EDAs. Even some EDA's performance that was previously thought inefficient can be improved surprisingly with the help of CMR. CMR can also guarantee those EDAs to be used with small scale of population (but still should be large enough to find the global optimum) to accelerate the convergence rate while maintaining the quality of solutions.
\end{abstract}

\section{INTRODUCTION}

Estimation of Distribution Algorithms (EDAs) [1], [2] have been studied intensively for their use in continuous optimization domains. Such approaches employ populationbased heuristic searching strategy. They are supposed a branch of evolutionary computation (EC). The main difference between EDAs and well-known Genetic Algorithms (GAs) [3], [4] is new population is generated with neither crossover nor mutation. Instead, new individuals are sampled from a probability distribution estimated from selected individuals of previous generations. The interrelations between variables are expressed explicitly in EDAs through joint probability distribution. Gaussian models are proposed in many existing continuous EDAs. In this paper, we classify Gaussian based EDAs into two categories in terms of what kind of covariance matrix is employed. As this paper shows, Gaussian based EDAs using full covariance matrix will fail in real data simulation under specific conditions caused by inevitable computation error. The appearance of ill-posed covariance matrix/negative variance is a universal problem that all Gaussian based EDAs using full covariance matrix suffer from. An effective technique named Covariance Matrix Repairing (CMR) is proposed to solve this problem. Experiments showed that CMR can significantly improve the robustness of those EDAs under any scale of population size. Especially for some EDA which is vulnerable to accumulated error, the performance can be surprisingly improved.

W. Dong is with the Key Laboratory for Complex Systems and Intelligence Science, Institute of Automation, Chinese Academy of Sciences, Beijing 100080, PR China (e-mail: weishan.dong@ia.ac.cn).

$X$. Yao is with The Centre of Excellence for Research in Computational Intelligence and Applications (CERCIA), School of Computer Science, The University of Birmingham, Birmingham B15 2TT, UK (e-mail: x.yao@cs.bham.ac.uk).
The remainder of this paper is organized as follows. In Section II, we present a review of Gaussian based continuous EDAs. Then we figure out the ill-posed covariance matrix and negative variance problem in section III. In section IV, we propose covariance matrix repairing and accumulated error removal on repairing the ill-posed covariance matrix, and outline the description of improved EDAs. Experimental studies are given in section $\mathrm{V}$ and our final conclusions are drawn in section VI.

\section{EDAs FOR CONTINUOUS GLOBAL Optimization}

EDAs were proposed originally for combinatory optimizations. Research on EDAs has been extended from discrete domain to continuous optimization and many progresses have been made. A survey of EDAs and their applications to continuous optimization can be found in [1], [5]-[7]. In this paper, we focus on continuous EDAs adopting Gaussian models, and divide them into two groups: Gaussian based EDAs using full covariance matrix, and Gaussian based EDAs using diagonal covariance matrix.

\section{A. Gaussian Based EDAs Using Full Covariance Matrix}

Such EDAs include: EMNA global, a variety of EMNA [1] which employs multivariate Gaussian density. Sample mean and covariance matrix are computed by maximum likelihood estimates; EMNA $\mathrm{a}_{\mathrm{a}}$ [1], an adaptive version of EMNA, whose flow is like a steady-state GA. Model parameters are updated in an incremental manner(see equations (3.34)-(3.35), pp.86, [1]). It was previously considered inefficient on benchmarks [1], but with the help of our new technique, considerable good performance can be achieved; RECEDA [8], which is essentially identical to EMNA, except the sampling method using Cholesky decomposition was explicitly outlined. Since EMNA is proposed earlier, and the sampling method RECEDA used is a common technique in random variate generation, RECEDA can be seen as a specific implementation of EMNA; EGNA, A class of algorithms ([9], [1], and [10]) based on learning and simulation of Gaussian network. The conditional variance used in sampling is computed by equations (2.35)-(2.40), pp.49 in [1], which can also be found as equations (13)-(15) in [10]; IDEA, a framework that different pdf (probabilistic density function) can be used within ([11], [12], and [6]). If normal pdf is used, sample mean and covariance matrix are estimated in the same way as in EMNA global. Conditional variance is computed by inverse of covariance matrix (see equation (50), pp.57, [11]). All EDAs above have a common characteristic of using essentially a multivariate Gaussian model, with a full covariance matrix. 


\section{B. Gaussian Based EDAs Using Diagonal Covariance Ma- trix}

Such EDAs include: SHCLVND [13], PBIL ${ }_{c}$ [14], $\mathrm{UMDA}_{\mathrm{c}}^{\mathrm{G}}[1]$ and EDA+DE [15]. Their common characteristic is only diagonal covariance matrix is used to express a univariate marginal Gaussian model.

\section{Gaussian Mixture Based EDAs}

Still, there exist EDAs using Gaussian mixture models, such as Adaptive Gaussian Mixture model (AMix, [16], [17]), Mixed IDEA using multivariate normal mixture [18], CEGNA and CEGDA [19]. The mixture components of AMix are univariate Gaussians whereas in Mixed IDEA, CEGNA and CEGDA, the components are multivariate Gaussians. So they can also be classified as one of the two categories above. In this paper, we concentrate on single Gaussian based EDAs and our conclusions can be easily extended to mixture models.

\section{Why AND When GAUSSIAN BASED EDAs FAIL}

\section{A. Ill-posed Covariance Matrix}

We take EMNA as an example to analyze the impact of ill-posed covariance matrix on multivariate Gaussian based EDAs. Theoretically, covariance matrix $\boldsymbol{\Sigma}$ is assured to be positive semi-definite by its definition. If we have a computer with infinite precision, then given any kinds of data, whenever we calculate covariance matrix we always get a positive-semi definite result. In most of the cases in real data simulation, $\boldsymbol{\Sigma}$ is positive definite. If $\exists i \in\{1, \ldots, n\}$, s.t. the $i^{\text {th }}$ row and the $i^{\text {th }}$ column of $\Sigma$ are all zeros, which means the searching in the $i^{\text {th }}$ dimension has been converged, then we can remove all the zeros to make up an $n-1$ dimension matrix, which is still positive definite. To sample new individuals from a given $\boldsymbol{\Sigma}$, we should first decompose it into a product of two matrices [20]. This requires the positive definiteness of $\boldsymbol{\Sigma}$. For symmetric positive definite matrix $\boldsymbol{\Sigma}$, we can always find a nonsingular matrix $\mathbf{H}$, s.t. $\mathbf{H} \mathbf{H}^{\mathrm{T}}=\mathbf{\Sigma}$. By using Cholesky decomposition, a unique nonsingular lower triangular matrix $\mathbf{H}$ can always be found. To sample a vector $\mathbf{Y}$ from $\mathcal{N}(\boldsymbol{\mu}, \boldsymbol{\Sigma})$, we should:

1) Decompose $\boldsymbol{\Sigma}$ to find $\mathbf{H}$.

2) Generate $n$ independent normal variates $X_{1}, \ldots, X_{n}$, where $X_{i} \sim \mathcal{N}(0,1), i=1, \ldots, n$.

3) Return $\mathbf{Y}=\boldsymbol{\mu}+\mathbf{H X}$.

But it is well-known that $\boldsymbol{\Sigma}$ sometimes could not be positive semi-definite (i.e. it has negative eigenvalue) because of finite precision computation. The computation error increases when computing with odd distributed samples, especially when sample size is relatively small to problem size. Suppose a $4 \mathrm{~d}$ problem and we have a population of 6 individuals. A multivariate Gaussian is to be modeled up by the best 3 shown below:

\begin{tabular}{c|cccc} 
& $x_{0}$ & $x_{1}$ & $x_{2}$ & $x_{3}$ \\
\hline No.1 & 1.469109 & -2.055897 & -1.598237 & -1.058821 \\
No.2 & -1.621055 & -1.211287 & -2.240671 & -0.175928 \\
No.3 & -2.847571 & -1.013135 & -2.568607 & -0.389777
\end{tabular}

Note the indices of variables are zero based. Covariance matrix can be calculated as:

$$
\boldsymbol{\Sigma}=\left(\begin{array}{rrrr}
3.2990 & -0.8171 & 0.7307 & -0.5949 \\
-0.8171 & 0.2044 & -0.1799 & 0.1557 \\
0.7307 & -0.1799 & 0.1624 & -0.1274 \\
-0.5949 & 0.1557 & -0.1274 & 0.1414
\end{array}\right)
$$

By checking eigenvalues of $\Sigma$, we can find a negative eigenvalue: -0.000027573139082 . With such non positive semi-definite $\boldsymbol{\Sigma}$, decomposition and sampling new individuals can't be successfully accomplished. This is a deadlock for EMNA/RECEDA, the algorithm thus will break down. We call such covariance matrix ill-posed if it has negative eigenvalue. Existing related literatures have no remarks about such exceptions, but if we properly repair the covariance matrix we can make EMNA/RECEDA robust approaches, and achieve considerably good results, especially for EMNA . Both EMNA $\mathrm{E}_{\mathrm{a}}$ and EMNA globaluse Gaussian models, so intuitively the performances between them should have no significant difference in orders of magnitude. However [1] showed opposite results that the performance of EMNA $\mathrm{a}_{\mathrm{a}}$ much worse than $\mathrm{EMNA}_{\text {global }}$ on all benchmarks with same parameters. Later we will prove such results did not exhibit the true capability of $\mathrm{EMNA}_{\mathrm{a}}$. With covariance matrix repairing, it even outperforms some newly developed EDAs. Ill-posed covariance matrix also tampers IDEA with normal density and may cause negative conditional variance as we will see.

\section{B. Negative Variance}

1) Negative Variance of EGNA: Whatever, the conditional variance of variables should be always non negative. Here we take 2 examples to illustrate the negative cases of EGNA.

Example 1: We again adopt the data of section A. The Gaussian network structure and parameters will be computed based on the 3 selected individuals. Suppose we used some learning method and finally got a learnt Gaussian network, or we had constructed a Gaussian network by apriori. The structure is depicted as Fig. 1(a). Linear coefficients between variables can be computed by equation (2.36), pp.49, [1]:

$$
\begin{array}{ll}
b_{10}=-3.996935 & \\
b_{20}=4.498462 & b_{23}=-0.784121 \\
b_{30}=-4.206342 & b_{31}=1.100663
\end{array}
$$

Sample mean $\boldsymbol{\mu}=(-0.9998,-1.427,-2.136,-0.5415)$, and covariance matrix is the same as equation (1). Negative value occurs when we compute conditional variance of $x_{1}$. The first term in equation (2.37), pp.49, [1] equals $\boldsymbol{\Sigma}(1,1)$. The second term equals $b_{31}$. From Fig. 1(a) we see $x_{1}$ only has $x_{3}$ as its parent, so the last term in the equation equals zero. Then the conditional variance of $x_{1}$ is:

$$
\hat{v}_{1}=0.2044-1.100663+20=-0.896263<0
$$




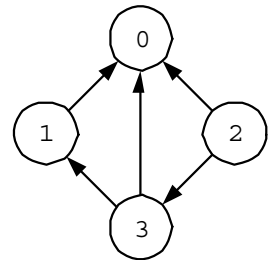

(a)

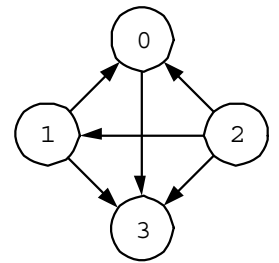

(b)
Fig. 1. Learnt structure of a Gaussian network. (a) is used in example 1 and (b) is used in example 2.

It is impossible to sample new data with a negative variance, also recall that $\boldsymbol{\Sigma}$ is not positive semi-definite. In other words, when $\Sigma$ is ill-posed, re-sampling new generation in EGNA can become iffy. But what will happen even if $\boldsymbol{\Sigma}$ is positive definite?

Example 2: Now we set sample size to a sufficient large number for a 4-dimensional problem and a more complicated Gaussian network structure has been learnt as Fig. 1(b). A positive definite covariance matrix is ${ }^{1}$ :

$$
\boldsymbol{\Sigma}=\left(\begin{array}{rrrr}
0.1197 & 0.01948 & 0.02699 & 0.03006 \\
0.01948 & 0.2362 & -0.0377 & -0.03477 \\
0.02699 & -0.0377 & 0.2419 & -0.03103 \\
0.03006 & -0.03477 & -0.03103 & 0.2434
\end{array}\right)
$$

And sample mean: $\boldsymbol{\mu}=(0.6047,0.3235,0.334,0.3483)$. The linear coefficients are:

$$
\begin{aligned}
& b_{03}=0.251251 \\
& b_{10}=0.082470
\end{aligned}
$$

Conditional variance of $x_{0}$ can be calculated as:

$$
\begin{aligned}
\hat{v}_{0}= & 0.1197-(0.082470+0.111572) \\
& +2 \times \frac{-0.0377 \times 0.01948 \times 0.02699}{0.2362 \times 0.2419} \\
= & -0.075081<0
\end{aligned}
$$

Now we see that even when $\boldsymbol{\Sigma}$ is positive definite, the calculation of conditional variance is not reliable. If there is no error in parameter estimating rules of EGNA, then there must be some exceptions like above that EGNA can not handle. Or we can say, EGNA deeply suffers from the computation error. Since unexpected randomly generated data constitute the essential optimum-searching function of EDAs, exceptions like above cannot be avoided in practice. Unfortunately, we haven't found a reasonable betterment to EGNA.

2) Negative Variance of IDEA: In order to sample new individual with normal pdf in IDEA, we should get the inverse of covariance matrix, $\boldsymbol{\Sigma}^{-1}$. The diagonal of $\boldsymbol{\Sigma}^{-1}$ is used in calculating conditional variances. If we simulate variable $x_{i}, i=0, \ldots, n-1$, the expression of variance $\tilde{\sigma}_{i}^{2}$

\footnotetext{
${ }^{1}$ This covariance matrix is simulated using EGNA (edge exclusion) on problem $F_{2}(\mathrm{x})$ which was presented in [9]. Parameters of EGNA were set to: population size $=4000$, selected size $=2000$, and dimension $n=4$.
}

(equation (50), pp.57, [11]) can be derived using theories of matrix:

$$
\tilde{\sigma}_{i}^{2}=\frac{1}{\boldsymbol{\Sigma}^{-1}(i, i)}=\frac{\operatorname{det} \boldsymbol{\Sigma}}{\mathbf{\Sigma}^{*}(i, i)}, i=0, \ldots, n-1 .
$$

Further more, we have the following conclusions:

1) For positive definite $\boldsymbol{\Sigma}$, we have $\operatorname{det} \boldsymbol{\Sigma}>0$, and $\boldsymbol{\Sigma}^{*}(i, i)>0, \tilde{\sigma}_{i}^{2}$ is always positive.

2) If $\boldsymbol{\Sigma}$ has negative eigenvalue(s), $\tilde{\sigma}_{i}^{2}$ can be negative.

For conclusion 2), suppose a $5 \times 5$ covariance matrix on a 5-dimensional problem ${ }^{2}$, which has a negative eigenvalue of pretty small absolute value of $-1.235541794400588 \mathrm{e}-17$ :

$$
\boldsymbol{\Sigma}=1 \mathrm{e}-11 \times\left(\begin{array}{rrrrr}
0.0061 & 0.0037 & -0.0011 & 0.0229 & 0.0590 \\
0.0037 & 0.0031 & -0.0013 & 0.0187 & 0.0487 \\
-0.0011 & -0.0013 & 0.0006 & -0.0075 & -0.0196 \\
0.0229 & 0.0187 & -0.0075 & 0.1125 & 0.2919 \\
0.0590 & 0.0487 & -0.0196 & 0.2919 & 0.7578
\end{array}\right)
$$

This is a nearly converged covariance matrix as we see. Thus we have:

$$
\boldsymbol{\Sigma}^{-1}=1 \mathrm{e}+17 \times\left(\begin{array}{rrrrr}
0.0387 & -0.4863 & -0.3006 & 0.0105 & 0.0164 \\
-0.4863 & 1.1753 & 2.2667 & -0.0886 & 0.0551 \\
-0.3006 & 2.2667 & 1.8863 & -0.0784 & -0.0433 \\
0.0105 & -0.0886 & -0.0784 & 0.0257 & -0.0071 \\
0.0164 & 0.0551 & -0.0433 & -0.0071 & -0.0032
\end{array}\right)
$$

We have $\boldsymbol{\Sigma}^{-1}(4,4)=-0.0032 \mathrm{e}+17<0$, thus $\tilde{\sigma}_{4}^{2}<0$. As we see, negative variance can also be observed in IDEA when ill-posed covariance matrix comes up. Conclusion 2) indicates that IDEA suffers from negative variance problem as well as EGNA.

In [21] and [22], Adaptive Variance Scaling (AVS) and Correlation Triggered AVS (CT-AVS) were proposed to use along with normal pdf in IDEA. The essential idea of AVS is to scale covariance matrix with an adaptive (also positive) coefficient $c^{\mathrm{AVS}}$ to help increase the area of exploration. The covariance matrix used for sampling is $c^{\mathrm{AVS}} \cdot \boldsymbol{\Sigma}$ instead of $\boldsymbol{\Sigma}$. With the knowledge of theories of matrix, we can see such scaling will not change the positive definiteness of $\boldsymbol{\Sigma}$. Neither AVS/CT-AVS could dodge negative variance.

As we see, no matter what techniques are used (covariance matrix decomposition or probability density factorization) in sampling stage, existing Gaussian based EDAs using full covariance matrix are all lack of resolvent to ill-posed covariance matrix. EGNA even fails in some cases when covariance matrix is not ill-posed. On the other hand, the diagonal of covariance matrix, which indicates the unconditional variances of variables, are always assured to be non negative. So in Gaussian based EDAs using diagonal covariance matrix, e.g. $\mathrm{UMDA}_{\mathrm{c}}^{\mathrm{G}}$, the situations discussed above can be totally avoided. But for some problems, the searching ability of full covariance matrix is much better than only diagonal. Resolving such a problem is necessary for those EDAs using full covariance matrix.

\footnotetext{
${ }^{2}$ This covariance matrix is simulated on 5-dimensional Rosenbrock gener-

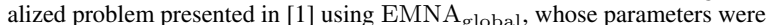
set to: population size $=20$, selected size $=10$. Although it was not simulated using IDEA, our aim is to illustrate that IDEA have difficulties to handle such covariance matrix.
} 
Actually, forming up factorized conditional densities through complicated transform made no enhancement in terms of searching ability of a Gaussian model. There is no significant evidence in practice so far that validates the priority of complicated probability model. Compared with sampling from factorization of normal pdf adopted by EGNA and IDEA, sampling through decomposition used in EMNA/RECEDA is more straightforward. Thus we favor the decomposition method in our experiments. But still we should do more to solve the remaining problem: How to tackle ill-posed covariance matrix?

\section{REPAIRING Ill-POSED COVARIANCE MATRIX: CMR}

A. Covariance Matrix Repairing and Accumulated Error Removal

In multivariate Gaussian based EDAs, covariance matrix $\boldsymbol{\Sigma}$ describes the shape of the learnt distribution. When illposed $\Sigma$ emerges, we should try to fix it so that searching can proceed. Ill-posed $\boldsymbol{\Sigma}$ is also a common problem in the experiments of statistical learning field. It is intrinsic flaw of finite-precision machines. In other fields, e.g. machine learning and pattern recognition, people usually add positive value to diagonal of $\boldsymbol{\Sigma}$ that may serves as regularization and leads to better estimate [23]. The more commonly used routine in statistical learning is to shrink $\boldsymbol{\Sigma}$ towards an identity matrix $I[24]$ to retain better generalized performance. There exists an optimal shrinking coefficient so that $\Sigma$ is not over-fitting the data meanwhile the estimate is not deviated too much. The more important thing is usually generalized performance rather than precision. But in EDA, any improper change to $\Sigma$ will destroy current density's statistical characteristics and affect the distributions of individuals in all the following generations. The iterative mechanism of EDA makes $\boldsymbol{\Sigma}$ more important role. Repairing $\boldsymbol{\Sigma}$ in EDA requires the precision most: Over-fitting is not a problem, but be required for convergence. Meanwhile regularization will make some already learnt characteristics of $\boldsymbol{\Sigma}$ lost or biased. So we not only want to repair $\boldsymbol{\Sigma}$ to positive semi-definite but also make least modifications to it to hold all learnt properties as much as possible. The sense of optimal here is based on the precision of estimate but not on the generalization performance as in machine learning.

Back to Gaussian based EDAs, we have a choice that to discard interactions of variables and do sampling just from the unconditional variances when $\boldsymbol{\Sigma}$ is ill-posed. It can be seen as a switch from multivariate Gaussian to univariate Gaussian. The algorithm's behavior thus will change. If we want to hold the searching ability of multivariate Gaussian all through, we have two other ways. One simple scheme is to re-sample current population until $\boldsymbol{\Sigma}$ is positive semidefinite. Such approach has obvious drawbacks: 1) We have to hold copies of mean and covariance matrix of previous generation. More storage space is required. 2) In order to calculate $\boldsymbol{\Sigma}$ anew, evaluate whole population and select best individuals should be done after re-sampling. It takes much time, especially for problems that need long time to evaluate.

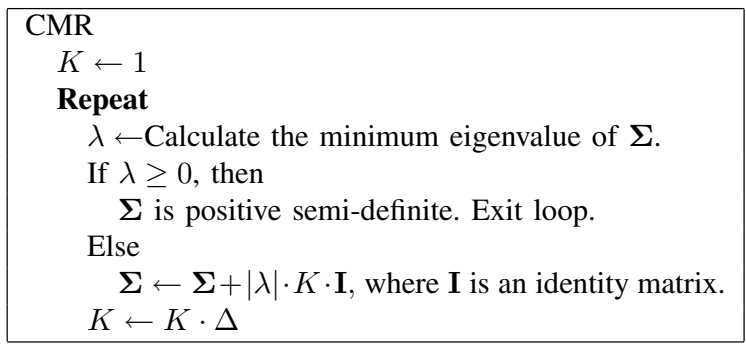

Fig. 2. Covariance Matrix Repairing (CMR).

3) Experimental observations showed that some problems have high probability to produce ill-posed $\boldsymbol{\Sigma}$, especially for small scale of population (see Section V), so limited times of retries can not be guaranteed.

Another way is back to add positive value to diagonal of $\boldsymbol{\Sigma}$. This approach does not have the drawbacks of the former one. Being different from that in machine learning, we repair the matrix and simultaneously make least modifications to preserve the characteristic of previously learnt distribution as much as possible. Especially in the converging stage of EDA, adding relatively big value can destroy nearly converged variance of a variable, and will disperse the sample points on that dimension in next generation. The value we chose is: The absolute value of the minimum eigenvalue $(<0$ if $\Sigma$ is ill-posed) of $\boldsymbol{\Sigma}$, multiplies a self-adapted positive coefficient $k$. The flow of Covariance Matrix Repairing (CMR) is shown in Fig. 2.

In practice, negative eigenvalue of ill-posed $\Sigma$ usually has pretty small absolute value, so diagonal of $\boldsymbol{\Sigma}$ will not be enlarged much. When calculating eigenvalues, precision of floating-point computation may get lost in the case of extremely small value of $|\lambda|$, thereby simply adding $|\lambda|$ may has no effect on changing the minimum eigenvalue at the precision level. That is why $K$ is introduced. The step size $\Delta$ which is set to a constant value $(>1.0)$ can help compensate the lost precision. For an $n$-dimensional problem, each loop of CMR costs $O\left(n^{3}\right)$ in eigenvalue decomposition. In our observations, with $\Delta=1.5$ almost all CMR routines can be terminated within 3 loops. The number of loops depends on the value of $\Delta$, and current computation error which is uncontrollable. Fortunately, the loop usually ends up with just one through without a second time repairing, having the time cost of $O\left(n^{3}\right)$ which only depends on problem size.

Additional operations should be done for EMNAabecause of its incremental updating rules. On finite-precision machines, accumulated error of $\boldsymbol{\mu}$ and $\boldsymbol{\Sigma}$ in EMNAakeeps growing as the searching proceed, and will be definitely much bigger than EMNA $_{\text {global }}$ in a long run, whereas EMNA $_{\text {global }}$ re-compute $\boldsymbol{\mu}$ and $\boldsymbol{\Sigma}$ thoroughly at every gener-

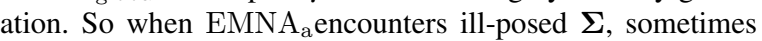
it is just caused by accumulated error. Re-compute $\boldsymbol{\mu}$ and $\boldsymbol{\Sigma}$ using the estimate rules of $\mathrm{EMNA}_{\text {global }}$ (equations (3.31)(3.33), pp.84, [1]) can remove the error and CMR can be called if $\Sigma$ is still ill-posed. In EMNA $_{\text {global, }}$ re-estimate 


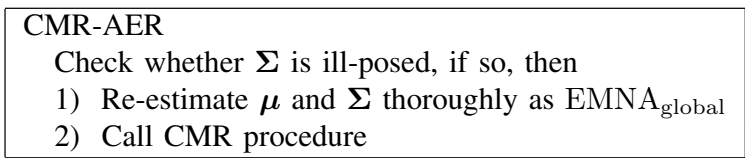

Fig. 3. CMR with Accumulated Error Removal (CMR-AER).

is a time-cost procedure which visits the whole population to gather information. Since EMNA $_{\text {globalgenerate a whole }}$ population from the estimated model, average time cost on each individual is acceptable. If we do the same in EMNA $_{a}$ at every generation, accumulated error can be reduced to trivial of course. But EMNA $\mathrm{E}_{\mathrm{a}}$ generates only one individual at a time, thus average time cost on each individual is too expensive. A compromise is to do re-estimate when necessary, and just estimate along with the incremental updating rules and ignore the accumulated error as long as $\boldsymbol{\Sigma}$ is not illposed. Experimental studies validated our thoughts. The flow of Covariance Matrix Repairing with Accumulated Error

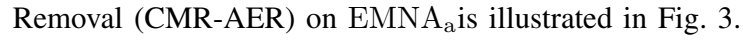

The main difference between CMR and previously mentioned AVS/CT-AVS is that CMR/CMR-AER focus on repairing ill-posed covariance matrix so as to improve algorithms' robustness, whereas AVS/CT-AVS ground on the positive definiteness of covariance matrix to improve the exploring effectiveness. It is possible to combine CMR with AVS/CT-AVS together to improve EDAs' performances. Of course if we add sufficient big value to the diagonal of covariance matrix we could also enhance the exploring effectiveness. But at the same time the precision of estimate is lost. In the following experiments, we can see previous results of EMNA may be trapped just in this way.

Evolution Strategy with Covariance Matrix Adaptation (CMA-ES) [25] is a well-known evolutionary algorithm that also adopts covariance matrix. However it does not suffer from ill-posed covariance matrix problem. The adaptation of covariance matrix in CMA-ES is to start from an identity matrix, then in every iteration it adapts by linear combination of itself and a positive definite matrix which is product of evolution path and its transpose (equation (3) of [25]). This guarantee the adapted covariance matrix is always positive definite. However, Gaussian based EDAs estimate covariance matrix from randomized samples, this introduces the risk of computation error on finite-precision computers. If the error exceeds some extent, the covariance matrix then become illposed. Precision plays the crucial role here.

So far as CMR could repair ill-posed covariance matrix, the negative variance problem of IDEA could be solved as well as those Gaussian based EDAs using full covariance matrix, but except for EGNA.

\section{B. EDA with CMR and CMR-AER}

Since all related EDAs suffer from the same problem, in this paper we only combine CMR with EMNA for demonstration. We name these algorithms as a class of algorithms EMNA-CMR. The pseudocodes for EMNA global-CMR and $^{-C M}$ EMNA $_{\mathrm{a}}$-CMR are shown in Fig. 4 and Fig. 5.

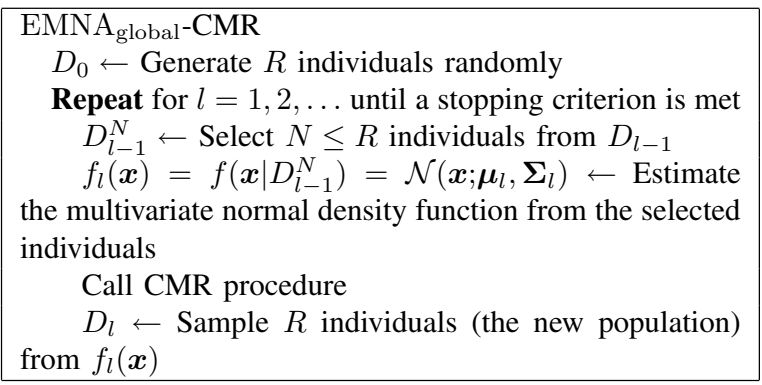

Fig. 4. Pseudocode for EMNA $_{\text {global-CMR. }}$

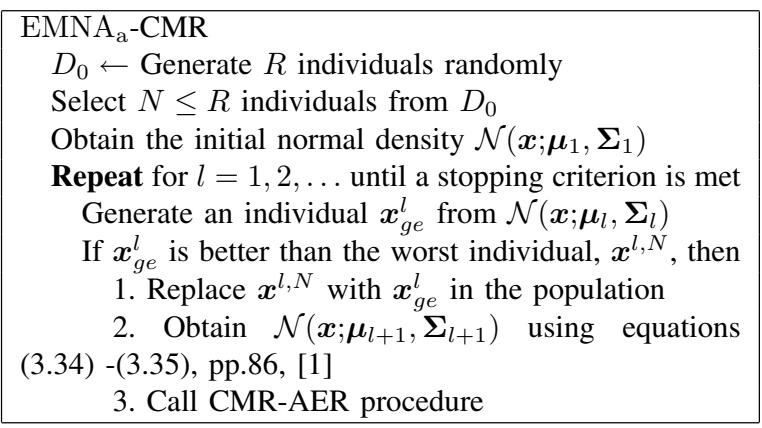

Fig. 5. Pseudocode for $\mathrm{EMNA}_{\mathrm{a}}$-CMR.

When extending to EDAs based on Gaussian mixture models, we can apply CMR/CMR-AER to each of the Gaussian component that uses full covariance matrix. If $N c$ is the number of Gaussian components using full covariance matrices, computational time of CMR is just $N c \times O\left(n^{3}\right)$, which is $N c$ times of that in single Gaussian based EDAs. It is linear scaled.

\section{EXPERIMENTAL STUDIES}

\section{A. Comparisons with EMNA Based Algorithms}

We present two comparisons here. The first one is between EMNA-CMR and original EMNA. The second one is between EMNA-CMR and EMNA-B [26]. In our observations, EMNA based algorithms without any repairs on covariance matrix will surely fail on problems that easily produce illposed covariance matrix. We are unable to obtain any replies from the authors of EMNA how those algorithms clear away such obstacles, so we cannot re-implement them exactly but to adopt previously published results.

1) EMNA-CMR vs. Original EMNA: The benchmarks and the results of original EMNA are from [1]. Five problems (SumCan, Griewangk, Sphere, Rosenbrock, and Ackley) with dimensions of 10 and 50 are involved. Details of these functions please see pp.182, [1]. We also adopted the same parameters in [1]: population size of 2000; the best 1000 are selected by truncation selection; elitist approach was used. The stop criterion is met when best fitness is within 1e-6 error of global optimal, or the number of evaluations reaches 301850, or the algorithm converges. The convergence justification was not claimed in [1], but it does seem that some stop 
TABLE I

COMPARISON BETWEEN EMNA-CMR AND ORIGINAL EMNA

\begin{tabular}{|c|c|c|c|c|}
\hline Function & Algorithm & Best fitness & No. Eval. & No. CMR \\
\hline \multirow{4}{*}{ SumCan-10d } & EMNA $_{\text {global }}$ & $99999.1 \pm 1.03 \mathrm{e}-01$ & $192904 \pm 1056.6$ & $\mathrm{~N} / \mathrm{A}$ \\
\hline & EMNA global $^{-\mathrm{C}}$ & $100000.0 \pm 2.51 \mathrm{e}-03$ & $301850 \pm 0.0$ & 0.0 \\
\hline & EMNA $_{a}$ & $6.40978 \pm 6.78 \mathrm{e}-01$ & $301850 \pm 0.0$ & N/A \\
\hline & EMNA $_{a}^{a}$-CMR & $100000.0 \pm 0.00$ & $149795 \pm 1612.6$ & 2.0 \\
\hline \multirow{2}{*}{ SumCan-50d } & EMNA $_{\text {global }}$ & $86190.7 \pm 1.31 \mathrm{e}+04$ & $301850 \pm 0.0$ & N/A \\
\hline & EMNA glo & $61729.1 \pm 3.42 \mathrm{e}+03$ & $301850 \pm 0.0$ & 0.0 \\
\hline \multirow{4}{*}{ Griewangk-10d } & EMNA glob & $\left.5.1166 \mathrm{e}-02 \pm 1.67 \mathrm{e}-02{ }^{*}\right)$ & $301850 \pm 0.0$ & $\mathrm{~N} / \mathrm{A}$ \\
\hline & $\mathrm{EMNA}_{g l o}$ & $7.4798 \mathrm{e}-07 \pm 1.68 \mathrm{e}-07$ & $121940 \pm 1660.1$ & 0.0 \\
\hline & EMNA $_{a}$ & $12.9407 \pm 3.43$ & $301850 \pm 0.0$ & $\mathrm{~N} / \mathrm{A}$ \\
\hline & EMNA $_{a}$-CMR & $7.9948 \mathrm{e}-07 \pm 1.50 \mathrm{e}-07$ & $142192 \pm 30614.8$ & 0.0 \\
\hline \multirow{2}{*}{ Griewangk-50d } & EMNA global & $8.7673 \mathrm{e}-06 \pm 1.03 \mathrm{e}-06$ & $216292 \pm 842.8$ & $\mathrm{~N} / \mathrm{A}$ \\
\hline & EMNA $g l o b$ & $8.6016 \mathrm{e}-07 \pm 9.40 \mathrm{e}-08$ & $274397 \pm 1478.6$ & 0.0 \\
\hline \multirow{4}{*}{ Sphere-10d } & EMNA global & $7.3350 \mathrm{e}-06 \pm 2.24 \mathrm{e}-06$ & $94353.8 \pm 842.8$ & $\mathrm{~N} / \mathrm{A}$ \\
\hline & EMNA $g l c$ & $7.1930 \mathrm{e}-07 \pm 1.80 \mathrm{e}-07$ & $122739.6 \pm 1447.2$ & 0.0 \\
\hline & EMNA $_{a}$ & $4.8107 \mathrm{e}+04 \pm 1.32 \mathrm{e}+04$ & $301000 \pm 0.0$ & N/A \\
\hline & EMNA $a-C M R_{a}$ & $7.5614 \mathrm{e}-07 \pm 1.93 \mathrm{e}-07$ & 44929. & 0.0 \\
\hline \multirow{2}{*}{ Sphere-50d } & EMNA global & $8.5225 \mathrm{e}-06 \pm 1.35 \mathrm{e}-06$ & $247477.2 \pm 1264.2$ & N/A \\
\hline & EMNA $g l o$ & $2.0156 \mathrm{e}-06 \pm 2.47 \mathrm{e}-07$ & $301850 \pm 0.0$ & 0.0 \\
\hline \multirow{4}{*}{ Rosenbrock-10d } & EMNA global & $8.7201 \pm 4.33 \mathrm{e}-02$ & $289056.4 \pm 40456.9$ & N/A \\
\hline & EMNA $g l o$ & $7.8217 \pm 1.99 \mathrm{e}-01$ & $301850 \pm 0.0$ & 0.0 \\
\hline & $\mathrm{EMNA}_{a}$ & $3263.0010 \pm 1216.75$ & $301000 \pm 0.0$ & N/A \\
\hline & $\mathrm{EMNA}_{a}-\mathrm{C}$ & $7.6078 \pm 2.22 \mathrm{e}-01$ & $297185.8 \pm 20719.9$ & 3.9 \\
\hline \multirow{2}{*}{ Rosenbrock-50d } & EMNA $g l o b$ & $49.7588 \pm 0.52$ & $296252.8 \pm 7287.1$ & N/A \\
\hline & EMNA $g l o b$ & $52.8836 \pm 2.42$ & $301850 \pm 0.0$ & 0.0 \\
\hline \multirow{4}{*}{ Ackley-10d } & EMNA global & $8.9265 \mathrm{e}-06 \pm 6.89 \mathrm{e}-07$ & $119141.4 \pm 1032.3$ & $\mathrm{~N} / \mathrm{A}$ \\
\hline & EMNA global $^{-\mathrm{CMR}}$ & $8.4348 \mathrm{e}-07 \pm 1.19 \mathrm{e}-07$ & $165185.0 \pm 1436.1$ & 0.0 \\
\hline & & & & \\
\hline & & $8.9449 \mathrm{e}-07 \pm 9.95 \mathrm{e}-08$ & $69518.2 \pm 960.7$ & 1.0 \\
\hline \multirow{2}{*}{ Ackley-50d } & EMNA glob & $9.5926 \mathrm{e}-06 \pm 2.39 \mathrm{e}-07$ & $291255.3 \pm 1349.2$ & $\mathrm{~N} / \mathrm{A}$ \\
\hline & EMNA $_{\text {global }}$ & $5.4979 \mathrm{e}-05 \pm 4.00 \mathrm{e}-06$ & $301850 \pm 0.0$ & 0.0 \\
\hline
\end{tabular}

* It seems to be an editorial error in [1]. The standard deviation value may be e-07.

criterion related to convergence was used, and some of the results seemed to be gained within 1e-5 error rather than

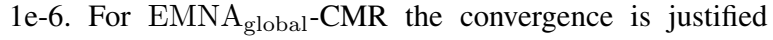
when average fitness of population between two neighbor generations is smaller than 1e-10. For $\mathrm{EMNA}_{\mathrm{a}}-\mathrm{CMR}$, if no improvement observed in continuous 1000 (equals the selected size) iterations, we consider it converged. Parameter $\Delta$ in CMR was set to 1.5 . Table I depicts the average performance of 30 independent runs of EMNA-CMR, while data of original EMNA was averaged on 10 runs. Mean values with standard deviations are provided. The performances of $\mathrm{EMNA}_{\mathrm{a}}$ on all 50d problems were not given in [1], so comparisons were not made either. When CMR/CMR-AER repairs covariance matrix, we call it an activation. Those repairs need more than one loop inside CMR were also counted as one activation. The mean times that CMR/CMRAER was activated during multiple runs are provided as well.

From the results we see all the runs of EMNA global-CMR did not activate CMR at all. EMNA global CMR outperforms EMNA $_{\text {global }}$ a little on Griewangk, whereas no significant difference for the rest. Meanwhile, EMNA $\mathrm{a}_{\mathrm{a}}$-CMR obviously outperforms $\mathrm{EMNA}_{\mathrm{a}}$ on all problems. Especially on SumCan, Rosenbrock and Ackley, with on average 1.0 to 2.1 activations of CMR-AER the best fitness values EMNA $\mathrm{a}_{\mathrm{a}}$-CMR achieved are far better than EMNA $_{\mathrm{a}}$ and indeed comparable to EMNA $_{\text {global }}$. EMNA EMR $_{\mathrm{a}} \mathrm{CM}$ even found the optimum with least evaluations on SumCan and Ackley, and obtained best solution on Rosenbrock with identical order of evaluations among all. However, on Griewangk and Sphere, CMRAER hadn't been activated, but EMNA $\mathrm{a}_{\mathrm{a}}$-CMR still achieved good result on Griewangk with limited evaluations, and spent least evaluations on Sphere to reach the optimum. The big difference may be caused by the neglect of accumulated error in original EMNA as we stressed before. Those non-zero activations of CMR-AER means that ill-posed covariance matrix indeed emerged, original EMNA should have broken down. It can be stated affirmatively that we found improper covariance matrix manipulations in previous implementation of EMNA. We guess original EMNA added relatively large values to the diagonal of covariance matrix: Slightly better results of original EMNA global $_{\text {appeared on those }}$ high dimensional problems need more exploration within limited evaluations, such as SumCan-50d, Rosenbrock-50d and Ackley-50d; While on low dimensional problems need more local search (estimate precision demanded) original EMNA performance worse. That may because the wrongly enlarged variances deteriorate the convergence rate. Whatsoever, CMR/CMR-AER help to show the real searching ability of Gaussian model. Although the number of activations of CMR-AER is small, it really make big difference and indeed help enhance the performance of EMNA EMNA $_{\mathrm{a}}$ CMR even outperforms EMNA global $_{\text {and }}$ EMNA $_{\text {global }}-\mathrm{CMR}$ on 10d problems including SumCan, Sphere, Rosenbrock, and Ackley. It is a reversal of previous results in [1], but it does verify our intuition and previous analysis.

2) EMNA-CMR vs. EMNA-B: EMNA-B [26] is a newly developed EMNA based algorithm using Boltzmann selection (B-Selection for short). The same function suite of experiment 1) with dimension of 10 was used again to show the robustness of EMNA-CMR on evolving small scale of population. The experimental setup was the same as 1) except the limit of evaluations was changed to 300000. Results adopted from [26] were evaluated after 10 runs. Population size of EMNA-B was 400, with selected size automatically determined during evolution. We assigned the same population size to EMNA-CMR, and still used truncation selection but with alternative selected sizes. Actually, the 'real' population size of EMNAain evolution is the selected size $N$. When $N$ is too small to establish an initial distribution well covering the problem, early convergence will deteriorate algorithm's stability. In other words, comparisons between EMNA $_{\text {global- }}{ }^{-}$ CMR and EMNA $\mathrm{a}_{\mathrm{a}}$-CMR under same small selected size are actually not fair. So we set different selected sizes to each of them on all the problems to examine their underlying searching abilities, and whether CMR functions well under varied parameter settings. Since the selected sizes we chose are all fewer than population size $(=400)$, comparisons with EMNA-B still make sense. Results of EMNA-CMR were evaluated after 100 runs. Table II exhibits the data. The best result of each algorithm (considering the mean best fitness value achieved, while preferring fewer evaluation times) on each problem is stressed in bold.

From the results, we clearly see whenever EMNA-B is able to find the optimum, so is EMNA-CMR. As long as the selected size is enough for solving a problem, EMNACMR always find the optimum with much fewer evaluations than EMNA-B. More surprisingly, EMNA-CMR spends nearly $1 / 10$ times of evaluations on Griewangk to obtain 
TABLE II

COMPARISON BETWEEN EMNA-CMR AND EMNA-B

\begin{tabular}{|c|c|c|c|c|c|}
\hline Function & Algorithm & Selected size & Best fitness & No. Eval. & No. CMR \\
\hline \multirow{11}{*}{ SumCan } & EMNA-B & B-Selection & $1.00000 \mathrm{e}+05 \pm 1.1 \mathrm{e}-07$ & $92520 \pm 840$ & N/A \\
\hline & \multirow{6}{*}{ EMNA $_{\text {global-CMR }}$} & 200 & $1.00000 \mathrm{e}+05 \pm 0.00$ & $68833 \pm 465.9$ & 0 \\
\hline & & 100 & $1.00000 \mathrm{e}+05 \pm 0.00$ & $42199 \pm 331.3$ & 0 \\
\hline & & 90 & $1.00000 \mathrm{e}+05 \pm 1.57 \mathrm{e}-03$ & $40033 \pm 354.4$ & 0 \\
\hline & & 80 & $1.00000 \mathrm{e}+05 \pm 1.12 \mathrm{e}-02$ & $38229 \pm 166.7$ & $\mathbf{0}$ \\
\hline & & 70 & $9.99999 \mathrm{e}+04 \pm 1.04 \mathrm{e}-01$ & $36693 \pm 303.4$ & 0.15 \\
\hline & & 60 & $9.81645 \mathrm{e}+04 \pm 1.14 \mathrm{e}+04$ & $54839 \pm 2655.2$ & 24.80 \\
\hline & \multirow{4}{*}{ EMNA $a-C M R^{-C M}$} & 400 & $1.00000 \mathrm{e}+05 \pm 0.00$ & $59202 \pm 979.3$ & 2.0 \\
\hline & & 300 & $1.00000 \mathrm{e}+05 \pm 1.57 \mathrm{e}-03$ & $44487 \pm 2678.1$ & 2.0 \\
\hline & & 250 & $1.00000 \mathrm{e}+05 \pm 2.13 \mathrm{e}-02$ & $37921 \pm 10949.7$ & 3.17 \\
\hline & & 200 & $9.785351 \mathrm{e}+04 \pm 1.18 \mathrm{e}+04$ & $67364 \pm 64530.3$ & 134.31 \\
\hline \multirow{11}{*}{ Griewangk } & EMNA-B & B-Selection & $7.4 \mathrm{e}-07 \pm 1.1 \mathrm{e}-07$ & $134000 \pm 47000$ & N/A \\
\hline & \multirow{6}{*}{ EMNA $_{\text {global-CMR }}$} & 200 & $7.355729 \mathrm{e}-07 \pm 1.67 \mathrm{e}-07$ & $23801 \pm 377.8$ & 0 \\
\hline & & 100 & $6.861266 \mathrm{e}-07 \pm 1.97 \mathrm{e}-07$ & $15351 \pm 1127.8$ & 0 \\
\hline & & 90 & $4.806266 \mathrm{e}-06 \pm 4.14 \mathrm{e}-05$ & $14976 \pm 1970.6$ & 0 \\
\hline & & 80 & $1.793993 \mathrm{e}-05 \pm 1.57 \mathrm{e}-04$ & $14588 \pm 2605.8$ & 0 \\
\hline & & 70 & $1.385388 \mathrm{e}-04 \pm 8.68 \mathrm{e}-04$ & $14166 \pm 2985.0$ & 0 \\
\hline & & 60 & $9.181267 \mathrm{e}-04 \pm 3.28 \mathrm{e}-03$ & $15379 \pm 4797.9$ & 0 \\
\hline & \multirow{4}{*}{ EMNA $_{a}$-CMR } & 400 & $8.329941 \mathrm{e}-07 \pm 1.32 \mathrm{e}-07$ & $46915 \pm 16085.2$ & 0 \\
\hline & & 300 & $8.146310 \mathrm{e}-07 \pm 1.44 \mathrm{e}-07$ & $33094 \pm 9959.2$ & 0 \\
\hline & & 250 & $9.031477 \mathrm{e}-04 \pm 9.02 \mathrm{e}-03$ & $26754 \pm 9679.2$ & 0 \\
\hline & & 200 & $3.291624 \mathrm{e}-03 \pm 2.44 \mathrm{e}-02$ & $20135 \pm 9171.5$ & 0.03 \\
\hline \multirow{11}{*}{ Sphere } & EMNA-B & B-Selection & $7.5 \mathrm{e}-07 \pm 2.1 \mathrm{e}-07$ & $35200 \pm 420$ & N/A \\
\hline & \multirow{6}{*}{ EMNA global $^{-\mathrm{CMR}}$} & 200 & $7.265598 \mathrm{e}-07 \pm 1.74 \mathrm{e}-07$ & $24041 \pm 317.7$ & 0 \\
\hline & & 100 & $6.166161 \mathrm{e}-07 \pm 2.06 \mathrm{e}-07$ & $15031 \pm 234.2$ & 0 \\
\hline & & 90 & $6.336102 \mathrm{e}-07 \pm 2.10 \mathrm{e}-07$ & $14309 \pm 247.3$ & 0 \\
\hline & & 80 & $6.456295 \mathrm{e}-07 \pm 2.04 \mathrm{e}-07$ & $13559 \pm 219.5$ & 0 \\
\hline & & 70 & $6.518929 \mathrm{e}-07 \pm 2.01 \mathrm{e}-07$ & $12841 \pm 229.5$ & 0 \\
\hline & & 60 & $5.849304 \mathrm{e}-05 \pm 5.73 \mathrm{e}-04$ & $12434 \pm 1731.1$ & 0 \\
\hline & \multirow{4}{*}{ EMNA $_{a}$-CMR } & 400 & $8.182929 \mathrm{e}-07 \pm 1.51 \mathrm{e}-07$ & $20426 \pm 299.6$ & 0 \\
\hline & & 300 & $8.096539 \mathrm{e}-07 \pm 1.54 \mathrm{e}-07$ & $15313 \pm 231.3$ & 0 \\
\hline & & 250 & $8.336055 \mathrm{e}-07 \pm 1.37 \mathrm{e}-07$ & $12752 \pm 213.8$ & 0 \\
\hline & & 200 & $2.235869 \mathrm{e}-06 \pm 1.15 \mathrm{e}-05$ & $12335 \pm 11766.1$ & 0.29 \\
\hline \multirow{11}{*}{ Rosenbrock } & EMNA-B & B-Selection & $6.33 \pm 0.37$ & $300400 \pm 0$ & N/A \\
\hline & \multirow{6}{*}{ EMNA global $^{\text {-CMR }}$} & 200 & $8.084920 \pm 5.02 \mathrm{e}-01$ & $53850 \pm 18600.0$ & 0 \\
\hline & & 100 & $7.741491 \pm 5.20 \mathrm{e}-01$ & $29292 \pm 4090.3$ & 0 \\
\hline & & 90 & $7.704431 \pm 5.46 \mathrm{e}-01$ & $27528 \pm 588.0$ & 0 \\
\hline & & 80 & $7.667069 \pm 6.19 \mathrm{e}-01$ & $26211 \pm 2639.8$ & 0 \\
\hline & & 70 & $7.749005 \pm 6.51 \mathrm{e}-01$ & $24962 \pm 2003.7$ & 0 \\
\hline & & 60 & $7.760831 \pm 6.90$ & $26347 \pm 27735.4$ & 2.21 \\
\hline & \multirow{4}{*}{ EMNA $_{a}$-CMR } & 400 & $7.692896 \pm 3.62 \mathrm{e}-01$ & $167927 \pm 61000.1$ & 7.99 \\
\hline & & 300 & $7.750415 \pm 4.38 \mathrm{e}-01$ & $99961 \pm 32413.2$ & 7.90 \\
\hline & & 250 & $7.853087 \pm 5.42 \mathrm{e}-01$ & $81644 \pm 23458.9$ & 9.31 \\
\hline & & 200 & $7.979813 \pm 5.54 \mathrm{e}-01$ & $64504 \pm 34056.2$ & 80.87 \\
\hline \multirow{11}{*}{ Ackley } & EMNA-B & B-Selection & $8.4 \mathrm{e}-07 \pm 1.0 \mathrm{e}-07$ & $43560 \pm 610$ & N/A \\
\hline & \multirow{6}{*}{ EMNA global $^{-\mathrm{CMR}}$} & 200 & $8.610074 \mathrm{e}-07 \pm 1.02 \mathrm{e}-07$ & $32192 \pm 371.4$ & 0 \\
\hline & & 100 & $8.045113 \mathrm{e}-07 \pm 1.36 \mathrm{e}-07$ & $20011 \pm 236.4$ & 0 \\
\hline & & 90 & $7.917779 \mathrm{e}-07 \pm 1.30 \mathrm{e}-07$ & $19021 \pm 254.0$ & 0 \\
\hline & & 80 & $7.725133 \mathrm{e}-07 \pm 1.42 \mathrm{e}-07$ & $18020 \pm 231.9$ & 0 \\
\hline & & 70 & $3.747483 \mathrm{e}-06 \pm 2.96 \mathrm{e}-05$ & $17102 \pm 846.9$ & 0 \\
\hline & & 60 & $4.700938 \mathrm{e}-04 \pm 3.42 \mathrm{e}-03$ & $16536 \pm 1682.6$ & 0 \\
\hline & \multirow{4}{*}{ EMNA $_{a}$-CMR } & 400 & $8.912338 \mathrm{e}-07 \pm 9.50 \mathrm{e}-08$ & $27399 \pm 388.5$ & 0.99 \\
\hline & & 300 & $9.111350 \mathrm{e}-07 \pm 8.96 \mathrm{e}-08$ & $20506 \pm 279.9$ & 0.99 \\
\hline & & 250 & $8.776402 \mathrm{e}-07 \pm 1.12 \mathrm{e}-07$ & $17085 \pm 249.2$ & 0.97 \\
\hline & & 200 & $8.855534 \mathrm{e}-05 \pm 5.81 \mathrm{e}-04$ & $18198 \pm 13681.7$ & 1.12 \\
\hline
\end{tabular}

the optimum on identical precision level. EMNA-B performs slightly better only on Rosenbrock with the expense of more evaluations. It is very probable that due to improper covariance matrix manipulations as original EMNA does, the effect of B-Selection become questionable. Generally, in most of the cases, with constant selected sizes, we achieve identical solutions with far more fewer evaluations. Considering the low dimension of all problems, the convergence rate of EMNA-B seems be slowed down as well as original EMNA. It maybe again caused by adding relatively large value onto diagonal of covariance matrix. After all, our results do not mean B-Selection is of no use, but show that EMNA-B is also lack of considerations on properly tuning covariance matrix. With CMR, these algorithms/operators could be more powerful.

We see the smaller the population is, the more probable ill-posed covariance matrix comes up. It is consistent with experienced conclusion that small sample size easily induce ill-posed covariance matrix. And $\mathrm{EMNA}_{\mathrm{a}}$ is more fragile because of accumulated error as we stated. CMR/CMRAER guaranteed robustness of EMNA and even helped
TABLE III

COMPARISONS OF FOUR GAUSSIAN BASED EDAS

\begin{tabular}{|c|c|c|c|c|c|}
\hline & & EMNA global $^{-\mathrm{CMR}}$ & 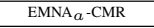 & EDA+DE & $\mathrm{UMDA}_{c}^{G}$ \\
\hline \multirow{3}{*}{$f_{1}$} & Best & $\begin{array}{c}8.625399 \mathrm{e}-07 \pm \\
8.78 \mathrm{e}-08\end{array}$ & $\begin{array}{c}8.838077 \mathrm{e}-07 \pm \\
1.01 \mathrm{e}-07\end{array}$ & $\begin{array}{c}9.285593 \mathrm{e}-04 \pm \\
2.10 \mathrm{e}-04\end{array}$ & $\begin{array}{c}8.614754 \mathrm{e}-07 \pm \\
1.05 \mathrm{e}-07\end{array}$ \\
\hline & No. Eval. & $100833.4 \pm 826.9$ & $85546.8 \pm 658.6$ & $300000.0 \pm 0.0$ & $107260.3 \pm 614.3$ \\
\hline & \begin{tabular}{|l|} 
No. $C M R$ \\
\end{tabular} & 0 & 0 & N/A & N/A \\
\hline \multirow{3}{*}{$f_{3}$} & Best & $\begin{array}{c}8.222809 \mathrm{e}-07 \pm \\
1.01 \mathrm{e}-07 \\
\end{array}$ & $\begin{array}{c}9.140343 \mathrm{e}-07 \pm \\
7.50 \mathrm{e}-08\end{array}$ & $\begin{array}{c}3.946647 e+01 \pm \\
8.86 \\
\end{array}$ & $\begin{array}{c}3.054380 \mathrm{e}+02 \pm \\
1.08 \mathrm{e}+02 \\
\end{array}$ \\
\hline & No. Eval. & $102964.6 \pm 943.5$ & $87459.6 \pm 737.8$ & $300000.0 \pm 0.0$ & $300700.0 \pm 0.0$ \\
\hline & No. CMR & 0 & 0 & N/A & N/A \\
\hline \multirow{3}{*}{$f_{5}$} & Best & $31.46288 \pm 2.38$ & $28.63699 \pm 8.11 \mathrm{e}-01$ & $27.38791 \pm 1.57 \mathrm{e}-01$ & $28.00946 \pm 2.79 \mathrm{e}-02$ \\
\hline & \begin{tabular}{|l|} 
No. Eval. \\
\end{tabular} & $300700.0 \pm 0.0$ & $300000.0 \pm 0.0$ & $300000.0 \pm 0.0$ & $300700.0 \pm 0.0$ \\
\hline & No. CMR & 1.1 & 3.73 & N/A & N/A \\
\hline \multirow[t]{3}{*}{$f_{8}$} & Best & $\begin{array}{c}-3.693336 \mathrm{e}+03 \pm \\
3.65 \mathrm{e}+02 \\
\end{array}$ & $\begin{array}{c}-4.187740 \mathrm{e}+03 \pm \\
2.81 \mathrm{e}+02 \\
\end{array}$ & $\begin{array}{c}-9.015122 \mathrm{e}+03 \pm \\
8.74 \mathrm{e}+02 \\
\end{array}$ & $\begin{array}{c}-5.944311 \mathrm{e}+03 \pm \\
2.65 \mathrm{e}+02 \\
\end{array}$ \\
\hline & \begin{tabular}{|l|} 
No. Eval. \\
\end{tabular} & $600400.0 \pm 0.0$ & $600000.0 \pm 0.0$ & $600000.0 \pm 0.0$ & $600400.0 \pm 0.0$ \\
\hline & No. CMR & 4.6 & 0 & N/A & N/A \\
\hline \multirow{3}{*}{$f_{13}$} & Best & $\begin{array}{c}5.447203 \mathrm{e}-02 \\
3.33 \mathrm{e}-02\end{array}$ & $\begin{array}{c}2.740194 \mathrm{e}-03 \pm \\
5.55 \mathrm{e}-03\end{array}$ & $\begin{array}{c}7.014277 \mathrm{e}-04 \pm \\
2.01 \mathrm{e}-04\end{array}$ & $\begin{array}{c}8.361740 \mathrm{e}-07 \pm \\
1.15 \mathrm{e}-07\end{array}$ \\
\hline & No. Eval. & $300700.0 \pm 0.0$ & $257084.1 \pm 87313.3$ & $300000.0 \pm 0.0$ & $102864.7 \pm 1065.1$ \\
\hline & \begin{tabular}{|l|} 
No. $C M R$ \\
\end{tabular} & 0.13 & 4.53 & N/A & N/A \\
\hline \multirow{3}{*}{$f_{21}$} & Best & $-9.967863 \pm 6.68 \mathrm{e}-01$ & $-10.15320 \pm 0.00$ & $-10.15320 \pm 1.25 \mathrm{e}-07$ & $-4.986098 \pm 2.37$ \\
\hline & No. Eval. & $89578.0 \pm 73286.6$ & $29321.4 \pm 1239.6$ & $54133.3 \pm 2012.6$ & $291808.9 \pm 48698.6$ \\
\hline & \begin{tabular}{|l|} 
No. $C M R$ \\
\end{tabular} & 0 & 0 & N/A & $\mathrm{N} / \mathrm{A}$ \\
\hline \multirow{3}{*}{$f_{22}$} & Best & $-10.40294 \pm 1.03 \mathrm{e}-05$ & $-10.40294 \pm 3.31 \mathrm{e}-07$ & $-10.40294 \pm 7.32 \mathrm{e}-06$ & $-10.39731 \pm 2.08 \mathrm{e}-02$ \\
\hline & \begin{tabular}{|l|} 
No. Eval. \\
\end{tabular} & $282251.8 \pm 70207.4$ & $300000.0 \pm 0.0$ & $291400.0 \pm 47104.1$ & $291475.9 \pm 50522.5$ \\
\hline & \begin{tabular}{|l|} 
No. CMR \\
\end{tabular} & $\begin{array}{l}0 \\
0\end{array}$ & 1.0 & N/A & $\mathrm{N} / \mathrm{A}$ \\
\hline \multirow{3}{*}{$f_{23}$} & \begin{tabular}{|l|} 
Best \\
\end{tabular} & $-10.53641 \pm 3.39 \mathrm{e}-06$ & $-10.53641 \pm 4.41 \mathrm{e}-06$ & $-10.53641 \pm 4.29 \mathrm{e}-06$ & $-10.53641 \pm 3.96 \mathrm{e}-06$ \\
\hline & \begin{tabular}{|l|} 
No. Eval. \\
\end{tabular} & $264336.4 \pm 94296.8$ & $226387.0 \pm 124160.6$ & $239966.7 \pm 110685.5$ & $245721.7 \pm 111837.1$ \\
\hline & \begin{tabular}{|l|} 
No. $C M R$ \\
\end{tabular} & & 0.73 & N/A & N/A \\
\hline
\end{tabular}

EMNA $_{\mathrm{a}}$-CMR gain the best performance on SumCan and Ackley. Compared to experiment 1), CMR was also activated in EMNA $_{\text {global-CMR on SumCan and Rosenbrock when }}$ population sizes were small. This validates the necessity of CMR. Generally speaking, EMNA-CMR showed robustness on any scale of population while without CMR/CMR-AER, ill-posed covariance matrix would definitely embarrass the evolution.

\section{B. Comparisons with Other Gaussian Based EDAs}

Twenty-three benchmark functions [27] were used here. No Free Lunch Theorem ([28], [29]) indicates that under certain assumptions no single search algorithm is best on average for all problems. We want to illustrate a general comparison between EMNA-CMR with full covariance matrix and EDAs with diagonal covariance matrix to justify the necessity of CMR. We chose some illustrative results here, include: $f_{1}, f_{3}, f_{5}, f_{8}, f_{13}, f_{21}, f_{22}$, and $f_{23}$. Details of these functions please see [27].

Four EDAs were compared: EMNA global-CMR, EMNA $_{\mathrm{a}}$ $\mathrm{CMR}, \mathrm{UMDA}_{\mathrm{c}}^{\mathrm{G}}$ and EDA+DE. In all experiments, population size $R=1000$, and selected size $N=500$ were used for $\mathrm{EMNA}_{\text {global-CMR, }} \mathrm{UMDA}_{\mathrm{c}}^{\mathrm{G}}$, and EDA+DE. Because the 'real' population size of EMNA $\mathrm{a}_{\mathrm{a}} \mathrm{CMR}$ during evolution is the selected size $N$, in order to compare algorithms under same scale of population, we set its $N=R=1000$. Elitist approach was used for all. Stop criterion was met when the best fitness was within 1e-6 error of global optimal, or 300000 evaluations reached. Only for $f_{8}$, the evaluation limit was 600000. No convergence justification was used. Extra parameters in EDA+DE are $F$ and $\delta$. The best settings of these parameters are problem dependent, so we adopted the frequently used value of $F=0.5$ and $\delta=0.9$ from [15] for all functions. 30 independent runs were evaluated for each algorithm. 
The results are summarized in Table III. We can see multivariate Gaussian model performs better on $f_{3}$ and $f_{21}$, while univariate Gaussian is more effective on $f_{8}$ and $f_{13}$. We also find that $f_{5}, f_{8}$, and $f_{13}$ easily produce ill-posed covariance matrix. Note EMNA global $^{-C M R}$ ought to be less affected by computation error than $\mathrm{EMNA}_{\mathrm{a}}-\mathrm{CMR}$, but it surprisingly needs more activation of CMR on $f_{8}$. Results of $f_{5}, f_{13}, f_{22}$ and $f_{23}$ are similar to our previous results (data stressed in bold). All above again showed the necessity of CMR during random sampling.

\section{CONCLUSIONS}

This paper points out the ill-posed covariance matrix/negative variance problem in Gaussian based EDAs using full covariance matrix. Through counter examples and theoretical analysis, the universality of such a problem has been proved. We propose Covariance Matrix Repairing to solve this problem and combine CMR with EMNA. From our experimental comparisons, it can be stated that CMR/CMRAER is good at repairing ill-posed covariance matrix. Even a small number of activations of CMR/CMR-AER can make a big difference on algorithms' performance. Especially, previously ignored details of EMNA are studied in depth and surprising results have been obtained. CMR significantly enhances the robustness of Gaussian based EDAs using full covariance matrix so that such algorithms can be applied with any population sizes to different optimization problems. This implies that such EDAs can be used with small population sizes (but still should be large enough to find the global optimum) to accelerate the convergence rate while maintaining the quality of solutions. Since all related EDAs suffer from the same problem, the idea of CMR can also be applied to all related EDAs, such as RECEDA, IDEA, and EDAs which adopt mixture models of multivariate Gaussian, like Mixed IDEA, CEGNA and CEGDA. Robust EDAs with CMR can be more widely used in continuous optimization domains. Future work includes applying CMR to other related EDAs and combining CMR with other techniques like AVS/CT-AVS, B-Selection, etc. Another interesting issue is the influence on convergence rate brought by CMR, to be investigated in-depth in the future.

\section{ACKNOWLEDGMENT}

This work is partially supported by the Chinese Academy of Sciences and the national 973 programme in China (2004CB318103) and a grant from the Chinese Academy of Sciences for Outstanding Young Scholars from Overseas (No. 2F03B01).

\section{REFERENCES}

[1] P. Larrañaga and J.A. Lozano, Estimation of Distribution Algorithms, A New Tool for Evolutionary Computation. Kluwer Academic Publishers, 2001.

[2] H. Mühlenbein and G. Paaß, From recombination of genes to the estimation of distributions. Binary parameters. In Parallel Problem Solving from Nature - PPSN IV. Lecture Notes in Computer Science 1411, pp.178-187, 1996.

[3] D.E. Goldberg, Genetic Algorithms in Search, Optimization, and Machine Learning. Addison-Wesley, Reading, Massachusetts, USA, 1989.
[4] J.H. Holland, Adaptation in Natural and Artificial Systems. The University of Michigan Press, Michigan, 1975.

[5] M. Pelikan, D. Goldberg, and F. Lobo, A Survey to Optimization by Building and Using Probabilistic Models. Computational Optimization and Applications, 21(1):5-20, 2002.

[6] P.A.N. Bosman and D. Thierens, Expanding From Discrete To Continuous Estimation Of Distribution Algorithms: The IDEA. In Parallel Problem Solving From Nature - PPSN VI, pp.767-776, Springer-Verlag, 2000.

[7] M.R. Gallagher and M. Frean, Population-Based Continuous Optimization, Probabilistic Modelling and Mean Shift. Evolutionary Computation, 13(1):29-42, 2005.

[8] T. Paul and H. Iba, Real-Coded Estimation of Distribution Algorithm. In Proc. 5th Metaheuristics Int. Conf., 2003, pp.61-66.

[9] P. Larrañaga, R. Etxeberria, J.A. Lozano, and J.M. Peña, Optimization in continuous domains by learning and simulation of Gaussian networks. GECCO 2000, pp.201-204.

[10] A. Mendiburu, J.A. Lozano, J. Miguel-Alonso, Parallel Implementation of EDAs Based on Probabilistic Graphical Models, IEEE Trans. Evol. Comput., Vol.9, No.4, pp.406-423, 2005.

[11] P.A.N. Bosman and D. Thierens, An Algorithmic Framework For Density Estimation Based Evolutionary Algorithms. Utrecht University technical report UU-CS-1999-46, 1999.

[12] P.A.N. Bosman and D. Thierens, Continuous Iterated Density Estimation Evolutionary Algorithms Within The IDEA Framework. In Proc. OBUPM Workshop at GECCO 2000, pp.197-200.

[13] S. Rudlof and M. Köppen, Stochastic hill climbing with learning by vectors of normal distributions. In Proc. 1st Online Workshop on Soft Computing (WSC1), Nagoya, Japan, 1996, pp.60-70.

[14] M. Sebag and A. Ducoulombier, Extending population-based incremental learning to continuous search spaces, In Parallel Problem Solving from Nature - PPSN V, pp.418-427, Springer-Verlag, Berlin, 1998.

[15] J. Sun, Q. Zhang and E. Tsang, EDA+DE: New Evolutionary Algorithm for Global Optimisation, Information Sciences, Vol.169, Issue 3-4, pp.249-262, 2005.

[16] M.R. Gallagher, M. Frean, and T. Downs, Real-valued evolutionary optimization using a flexible probability density estimator. GECCO 1999, pp.840-846.

[17] M.R. Gallagher, Multi-layer perceptron error surfaces: Visualizations, structure and modeling. Technical Report Doctoral Thesis, Department of Computer Science and Electrical Engineering, University of Queensland, 2000.

[18] P.A.N. Bosman and D. Thierens, Mixed IDEAs. Technical Report UUCS-2000-45, Utrech University, 2000.

[19] Q. Lu and X. Yao, Clustering and Learning Gaussian Distribution for Continuous Optimization, IEEE Trans. Systems, Man, and Cybernetics, Part C, 35(2):195-204, May 2005.

[20] L. Devroye, Non-Uniform Random Variate Generation. New York: Springer-Verlag, 1986

[21] P.A.N. Bosman and J. Grahl, Matching Inductive Search Bias and Problem Structure in Continuous Estimation-of-Distribution Algorithms. Technical Report 03/2005, Dept. of Logistics, Mannheim Business School, 2005.

[22] J. Grahl, P.A.N. Bosman and F. Rothlauf, The Correlation-Triggered Adaptive Variance Scaling IDEA. GECCO 2006: 397-404.

[23] V. Vapnik, Statistical Learning Theory. New York: John Wiley \& Sons, 1998.

[24] O. Ledoit and M. Wolf, A well-conditioned estimator for largedimensional covariance matrices. J. Multivar. Anal.,vol.88, no.2, pp.365-411, 2004.

[25] N. Hansen and A. Ostermeier, Adapting Arbitrary Normal Mutation Distributions in Evolution Strategies: The Covariance Matrix Adaptation. In Proc. 1996 IEEE Int. Conf. on Evolutionary Computation: 312-317.

[26] Y. Cai, X. Sun, P. Jia, Probabilistic modeling for continuous EDA with Boltzmann selection and Kullback-Leibeler divergence. GECCO 2006: 389-396.

[27] X. Yao, Y. Liu and G. Lin, Evolutionary Programming Made Faster, IEEE Trans. Evol. Comput., Vol.3, No.2, pp.82-102, 1999.

[28] D.H. Wolpert and W.G. Macready, No Free Lunch Theorems for Search, Technical Report SFI-TR-95-02-010(Santa Fe Institute), 1995.

[29] D.H. Wolpert and W.G. Macready, No Free Lunch Theorems for Optimization, IEEE Trans. Evol. Comput., vol.1, pp.67-82, Apr. 1997. 